\title{
Orai1: A New Therapeutic Target for the Acute Kidney Injury-to-Chronic Kidney Disease Transition
}

\author{
David P. Basile Jason A. Collett \\ Department of Anatomy, Cell Biology \& Physiology, Indiana University of Medicine, Indianapolis, IN, USA
}

\section{Keywords}

Acute renal injury $\cdot$ CKD $\cdot$ Inflammation

\begin{abstract}
This review focuses on the potential mediation in the acute kidney injury (AKI)-to-chronic kidney disease (CKD) transition by lymphocytes. We highlight evidence that lymphocytes, particularly Th17 cells, modulate the severity of both acute injury and chronic kidney disease. Th17 cells are strongly influenced by the activity of the store-operated $\mathrm{Ca}^{2+}$ channel Orai1, which is upregulated on lymphocytes in animal models of AKI. Inhibition of this channel attenuates both acute and chronic kidney injury in rodent models. In addition, Oria1+ cells are increased in peripheral blood of patients with AKI. Similarly, peripheral blood cells manifest an early and sustained increase in Orai 1 expression in a rat
\end{abstract}

Contribution from the AKI and CRRT 2021 Symposium at the 26th International Conference on Advances in Critical Care Nephrology, A Virtual/Hybrid Event from San Diego, CA, USA, February 28-March 5, 2021. This symposium was supported in part by the NIDDK funded University of Alabama at Birmingham-University of California San Diego O'Brien Center for Acute Kidney Injury Research (P30DK079337).

karger@karger.com

(c) 2021 S. Karger AG, Basel

www.karger.com/nef

Karger" model of ischemia/reperfusion, suggesting that blood cell Orai1 may represent a marker informing potential Th17 activity in the setting of AKI or the AKI-to-CKD transition.

(c) 2021 S. Karger AG, Basel

Acute kidney injury (AKI) is associated with compromised renal function often due to damage from ischemia or nephrotoxicity. While AKI is associated with high mortality rates, surviving patients typically experience recovery of renal function resulting from efficient repair programs. However, not all surviving patients recover full renal function and frequently many patients may transition to chronic kidney disease (CKD). Evidence derived from studies in animal models in the last 20 years has identified multiple cellular mechanisms which may contribute to the predisposition to CKD and renal fibrosis after the apparent recovery from AKI. Such mechanisms may include rarefaction of renal capillaries and alterations of hemodynamic responses, which may exacerbate hypoxia, and impair sodium regulation leading to hypertension [1]. Other investigators have suggested that incomplete repair of epithelial cells may liberate a host of pro-fibrotic factors that affect activated myofibroblasts 


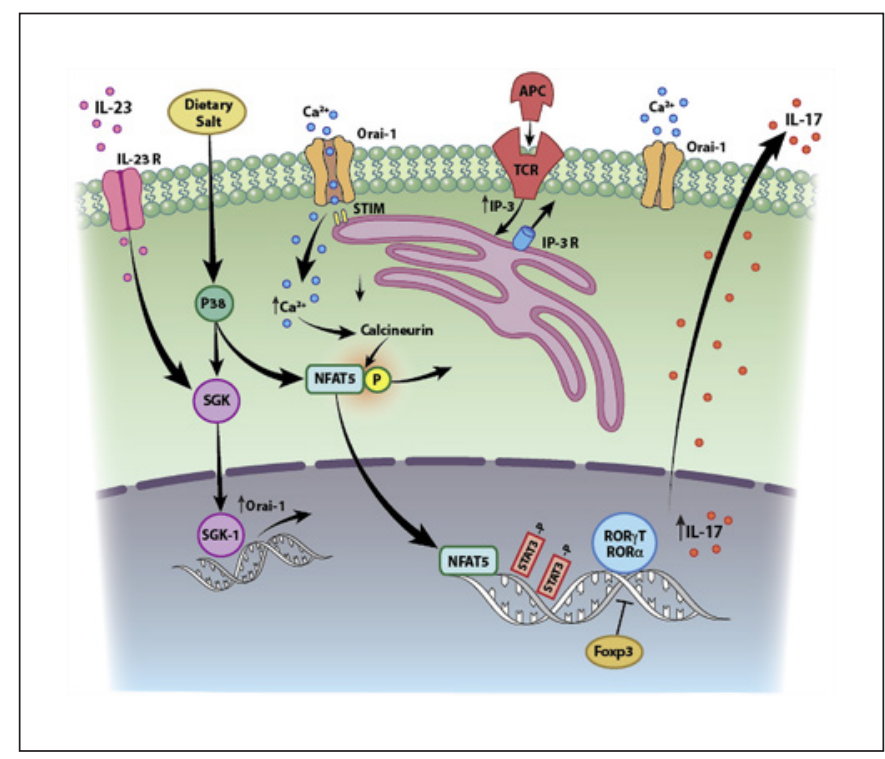

Fig. 1. Activation and differentiation of Th17 cells in the setting of renal injury and potential influence of salt. Th17 cell differentiation and IL17 activation occur secondary to TCR activation. Activation of STAT3 is required for induction of the transcription factor ROR $\gamma \mathrm{T}$, which mediates transcription of the signature cytokine, IL17. The process appears to be dependent on activation of the SOCC Orai1, which is initially activated by ER depletion of $\mathrm{Ca}^{2+}$, which is sensed by STIM1. The sustained elevation of Ca2+ mediated by Orail activates NFAT dephosphorylation and further drives IL17 expression. Orail is also activated by a high salt environment, possibly resulting from SGK signaling, which may further amplify Th17 activity in the setting of elevated dietary sodium intake. Reprinted from reference [7], with permission of the Korean Society of Nephrology.

contributing to the development of interstitial fibrosis [2, 3].

The immune system is known to modulate the development of AKI [4]. Previous studies from multiple groups have demonstrated an increase in T-lymphocyte infiltration in the setting of human AKI as well as in rodent models of AKI induced by ischemia reperfusion (I/R) [4]. Studies from Hamid Rabb's laboratory demonstrated persistent renal T-lymphocyte expression for several weeks following severe AKI and that adoptive transfer of splenocytes from post-AKI mice promoted proteinuria in naïve recipient mice [5]. In addition, work from our laboratory suggested that exposure to high salt diet, 5 weeks following recovery from AKI, enhanced renal inflammation, and CKD progression. The lymphocyte inhibitory agent, mycophenolate, abrogated the development of inflammation, fibrosis, and hypertension in this model [6].
Taken together, these reports suggest an important role of lymphocytes in the AKI-to-CKD transition.

The regulation of lymphocyte activation and differentiation is fundamental to understanding AKI and the AKI-to-CKD transition. T-helper 17 cells (Th17) are CD4+ lymphocytes characterized by the production of the cytokine IL17. Th17 cells are prominently expressed in autoimmune diseases, and several studies have demonstrated an increase in IL17 or Th17 cells in a variety of different kidney diseases [7]. IL17 is a highly pro-inflammatory cytokine with the potential to cause tissue damage by the promotion of neutrophil or macrophage infiltration. IL17 can also be liberated by other immune cell types such as NKT cells [7].

Accumulating evidence suggests that Th17 cells are a major differentiated T-helper subtype in models of AKI $[7,8]$. Following the apparent recovery of renal function following I/R-induced AKI, Th17 cells were prominently re-induced in kidney when rats were exposed to high-salt diet. The effect of high-salt diet to enhance Th17 cell activation is a commonly reported feature in models of autoimmune disease, which is thought to be due to the activation of the SGK1 and NFAT5 pathways [7] (Fig. 1). Inhibition of IL17 activity with an IL17RA-receptor antagonist, concurrent with elevated dietary salt treatment, attenuated the development of renal fibrosis and the infiltration of macrophages and neutrophils, suggesting an important role in the AKI-to-CKD transition [9]. IL17 may effect renal tubular cells to influence the production of other proinflammatory factors and may promote endothelial activity leading to inflammation or vasoconstriction [7].

Recently, we sought to understand the intracellular signaling pathways mediating Th17 cell activity in AKI and CKD. Previous studies have demonstrated that the store-operated $\mathrm{Ca}^{2+}$ channel (SOCC) Orail may mediate lymphocyte calcium signaling during differentiation. Orail is a transmembrane protein whose activity is regulated by levels of $\mathrm{ER} \mathrm{Ca}^{2+}$ stores via interaction with the $\mathrm{Ca}^{2+}$ sensing receptor Stromal Interaction Molecule 1(STIM1) in the ER membrane [10]. Upon interaction with the antigen presenting cells, the T-cell receptor triggers $\mathrm{ER} \mathrm{Ca}^{2+}$ release via activation of inositol triphosphate signaling. If ER $\mathrm{Ca}^{2+}$ stores are depleted, STIM1 directly interacts with Orail to induce conformational changes and promote influx of extracellular $\mathrm{Ca}^{2+}$ (Fig. 1). This results in sustained influx of $\mathrm{Ca}^{2+}$ which is thought to favor Th17 differentiation preferentially versus activation of T-helper 1 or T-helper 2 cells. Impairment of either Orail or Stim 1 has been shown to result in a severe combined immunodeficiency $[10,11]$. 
Our laboratory sought to determine the potential role of Orail in the activation of Th17 cells in the setting of AKI. Using flow-cytometry, kidney I/R injury increased the number of CD4+ cells that express the Orail channel as well as the number CD4+ cells expressing both Orai1 and IL17. Interestingly, IL17 expression in CD4 cells appeared to be dependent on the presence of the channel, since no IL17 was detected in cells which did not express the channel [12].

To further assess the involvement of Orail in Th17 activation, an in vitro assay was developed in which kidney CD4+ cells primed by AKI were stimulated in vitro with a combination of Ang II and elevated extracellular sodium. Under these conditions, CD4 cells derived from injured kidney increased IL17 expression, while CD4 cells from sham-controls did not. Inclusion of antagonists against Orai1 (BTP2/YM58483) or STIM1 (AnCoA4) inhibited expression of IL17 from AKI-primed CD4 cells in vitro [12].

Blockade of the SOCC was evaluated in AKI by administering YM58483 to rats. In acute studies, treatment with this antagonist significantly attenuated the development of AKI $24 \mathrm{~h}$ following I/R or glycerol induced rhabdomyolysis as determined by serum creatinine, tubular injury, and renal KIM-1 expression. Importantly, YM58483 significantly attenuated the influx of IL-17 expressing cells as well as the influx of neutrophils and macrophages but had little effect on the expression of Th1 or Th2 cells [12].

Whether Orail participates in the AKI-to-CKD transition was evaluated by subjecting post-I/R rats to a highsalt diet. Rats were treated continuously for 4 weeks during the high-salt diet phase with YM58483, which significantly attenuated the activation of Th17 cells as well as total CD4 and CD8 cells and total macrophages, proteinuria, and interstitial fibrosis. Moreover, YM58483 blocked the reduction in creatinine clearance relative to AKI rats treated with vehicle [12].

Taken together, these observations suggest that Orai1 may represent a target in AKI and the AKI-to-CKD transition. Importantly, this pathway seems to be activated in human AKI. Using peripheral blood cells, we demonstrated an increase in total blood cells and CD4+ cells expressing both Orail and IL17 in ICU patients following diagnosis of AKI [12]. We propose that peripheral blood cell activity of Orail may represent a marker of potential Th17 activity in early AKI or predisposition to CKD. To evaluate this possibility, we employed the widely utilized model of ischemia/reperfusion injury in Sprague-Dawley rats [12]. In pilot studies, we conducted FACS analysis on total blood cells from rats within $6 \mathrm{~h}$ of ischemia/reperfu-

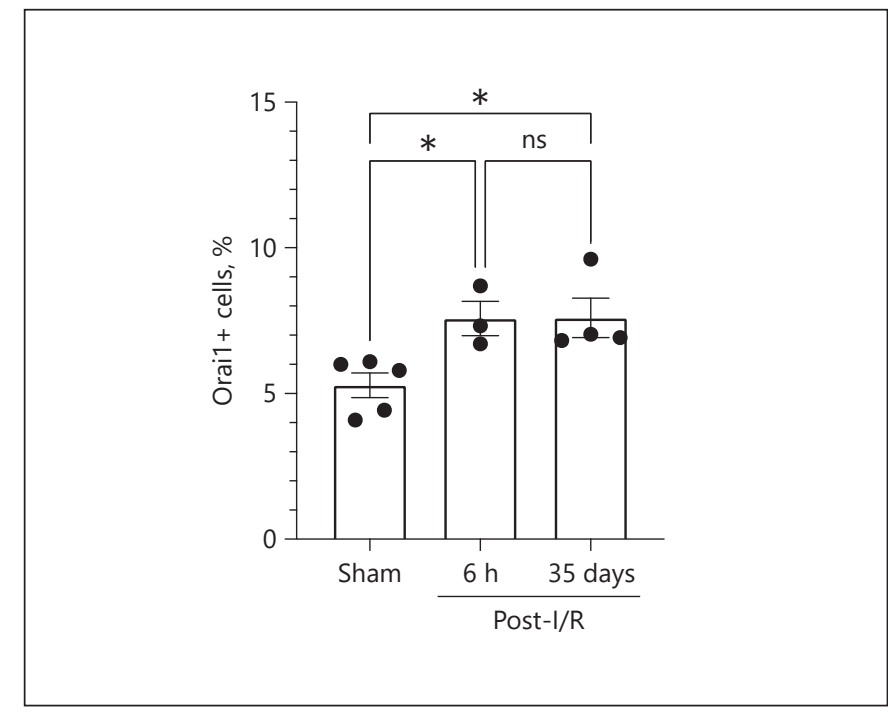

Fig. 2. Rapid and sustained increase in Orail expression in peripheral blood of rats. Sprague-Dawley ( 250 to $300 \mathrm{~g}$ ) rats were subjected to $40 \mathrm{~min}$ of bilateral renal ischemia and reperfusion for $6 \mathrm{~h}$ or $40 \mathrm{~min}$ of unilateral renal ischemia and reperfusion for 35 days. Total peripheral blood cells were stained with anti Orail antibodies similar to procedure described in reference [12] and subjected to FACS analysis. Data are expressed as \% Orail+ cells. I/R caused a significant increase in Orail+ cells both $6 \mathrm{~h}$ and 35 days after ischemia (*indicates $p<0.05$ vs. sham by ANOVA and Tukey's post hoc test).

sion injury. This early time point occurs prior to the manifestation of overt tubular damage but had a modest increase in plasma creatinine $(0.5 \mathrm{mg} / \mathrm{dL}$ vs. $1.6 \mathrm{mg} / \mathrm{dL} ; p<$ $0.05)$. A significant increase in the percentage of Orai1+ cells was observed within $6 \mathrm{~h}$ of I/R relative to sham (Fig. 2), suggesting that peripheral blood may provide an early accessible window into the activation state of the Orail pathway in immune cells during development of AKI.

We were also interested in determining if blood Orail activity may reflect the potential predisposition to progression following recovery from AKI. For this reason, we sampled blood from rats at 35 days following unilateral I/R. This time point and model are significant for 2 reasons: (1) there is typically minimal evidence of fibrosis at this time point and (2) exposure of rats to high-salt diet at this time induces a robust Th17 response associated with progression $[8,9,12]$. We observed a sustained increase in Orai $1+$ cells 35-days following recovery from I/R, suggesting blood cells may provide a useful approach to assess increased risk of CKD progression. We propose that future studies may be geared toward inves- 
tigating peripheral blood cell Orail expression in patients as a potential early marker of AKI or susceptibility to CKD.

Whether the beneficial effect of Orail blockade is mediated exclusively through inhibition of IL17 is not yet clear. In acute studies, the inclusion of an IL17 antagonist resulted in no additional protection beyond the effect of YM58483 alone, suggesting the primary effect was via IL17 [12]. Nevertheless, Mai et al. [13] reported that Orai1 is expressed in renal tubule cells and inhibition of the channel could prevent the process of epithelial-mesenchymal transition leading to renal fibrosis. In addition, given the importance of vascular regulation in the setting of $\mathrm{AKI}$ and $\mathrm{CKD}$, it is possible that Orail activity in endothelial or smooth muscle cells may influence AKI. An interesting study by Gandhirajan et al. [14] utilized an endothelial cell-specific knockout of STIM and demonstrated these mice were resistant to LPS induced lung injury. Whether inhibition of the endothelial STIM1/Orai1 pathway protects against kidney injury and whether such activity influences inflammation and/or hemodynamics has yet to be addressed.

Given the importance of SOCC in immune function as well as vascular and fibrotic processes, we propose that Orail represents a reasonable therapeutic target to attenuate either AKI or AKI-to-CKD. It is worth noting that several SOCC antagonists are under development and have been evaluated in Phase I trials for other indications [15]. If proven safe, the application of these agents could be envisioned for treatment of patients at high risk for $\mathrm{AKI}$ or to help minimize progression to CKD.

\section{Statement of Ethics}

Previously unpublished experiments described were approved by the Indiana University Institutional Animal Care and Use Committee.

\section{Conflict of Interest Statement}

The authors have no conflicts of interest to disclose.

\section{Funding Sources}

Work from the authors presented in this paper was supported by NIH Grant DK-063114 and the Indiana University Showalter Foundation.

\section{Author Contributions}

D.P.B. conceived, wrote, and edited the manuscript. J.A.C. conducted experiments, analyzed data, assembled figures, and edited the manuscript.

\section{References}

1 Ullah MM, Basile DP. Role of renal hypoxia in the progression from acute kidney injury to chronic kidney disease. Semin Nephrol. 2019; 39(6):567-80.

2 Basile DP, Bonventre JV, Mehta R, Nangaku $\mathrm{M}$, Unwin R, Rosner MH, et al. Progression after AKI: understanding maladaptive repair processes to predict and identify therapeutic treatments. J Am Soc Nephrol. 2016;27:68797.

3 Ferenbach DA, Bonventre JV. Mechanisms of maladaptive repair after AKI leading to accelerated kidney ageing and CKD. Nat Rev Nephrol. 2015;11:264-76.

4 Gharaie Fathabad S, Kurzhagen JT, Sadasivam M, Noel S, Bush E, Hamad ARA, et al. T lymphocytes in acute kidney injury and repair. Semin Nephrol. 2020;40(2):114-25.

5 Burne-Taney MJ, Liu M, Ascon D, Molls RR, Racusen L, Rabb H. Transfer of lymphocytes from mice with renal ischemia can induce albuminuria in naive mice: a possible mechanism linking early injury and progressive renal disease? Am J Physiol Renal Physiol. 2006; 291:F981-6.
6 Pechman KR, Basile DP, Lund H, Mattson DL. Immune suppression blocks sodium-sensitive hypertension following recovery from ischemic acute renal failure. Am J Physiol Regul Integr Comp Physiol. 2008;294:R12349.

7 Basile DP, Ullah MM, Collet JA, Mehrotra P. $\mathrm{T}$ helper 17 cells in the pathophysiology of acute and chronic kidney disease. Kidney Res Clin Pract. 2021;40(1):12-28.

8 Mehrotra P, Patel JB, Ivancic CM, Collett JA, Basile DP. Th-17 cell activation in response to high salt following acute kidney injury is associated with progressive fibrosis and attenuated by AT-1R antagonism. Kidney Int. 2015; 88:776-84.

9 Mehrotra P, Collett JA, McKinney S, Stevens J, Ivancic CM, Basile DP. IL-17 mediates neutrophil infiltration and renal fibrosis following recovery from ischemia reperfusion: compensatory role of natural killer cells in athymic rats. Am J Physiol Renal Physiol. 2017; 312:F385-97.
10 Derler I, Jardin I, Romanin C. Molecular mechanisms of STIM/Orai communication. Am J Physiol Cell Physiol. 2016;310:C643-62.

11 Feske S, Picard C, Fischer A. Immunodeficiency due to mutations in Orail and stim1. Clin Immunol. 2010;135:169-82.

12 Mehrotra P, Sturek M, Neyra JA, Basile DP. Calcium channel Orail promotes lymphocyte IL-17 expression and progressive kidney injury. J Clin Invest. 2019;129:4951-61.

13 Mai X, Shang J, Liang S, Yu B, Yuan J, Lin Y, et al. Blockade of Orail store-operated calcium entry protects against renal fibrosis. J Am Soc Nephrol. 2016;27:3063-78.

14 Gandhirajan RK, Meng S, Chandramoorthy HC, Mallilankaraman K, Mancarella S, Gao $\mathrm{H}$, et al. Blockade of Nox 2 and Stim 1 signaling limits lipopolysaccharide-induced vascular inflammation. J Clin Invest. 2013;123:887902.

15 Tian C, Du L, Zhou Y, Li M. Store-operated CRAC-channel inhibitors: opportunities and challenges. Fut Med Chem. 2016;8:817-32. 